\title{
Diffeomorphisms with Shadowable Measures
}

\author{
Kazumine Moriyasu ${ }^{1}$, Kazuhiro Sakai ${ }^{2, *}$ and Naoya Sumi ${ }^{3}$ \\ 1 Department of Mathematics, Tokushima University, Tokushima 770-8502, Japan; \\ moriyasu@tokushima-u.ac.jp \\ 2 Department of Mathematics, Utsunomiya University, Utsunomiya 321-8505, Japan \\ 3 Department of Mathematics, Kumamoto University, Kumamoto 860-8555, Japan; sumi@kumamoto-u.ac.jp \\ * Correspondence: kazsakai@cc.utsunomiya-u.ac.jp
}

Received: 8 November 2018; Accepted: 5 December 2018; Published: 7 December 2018

\begin{abstract}
In this paper, the notion of shadowable measures is introduced as a generalization of the shadowing property from the measure theoretical view point, and the set of diffeomorphisms satisfying the notion is considered. The dynamics of the $C^{1}$-interior of the set of diffeomorphisms possessing the shadowable measures is characterized as the uniform hyperbolicity.
\end{abstract}

Keywords: shadowing property; pseudo-orbit; shadowable measure; Axiom A; no-cycle condition; strong transversality condition

\section{Introduction}

The notion of pseudo-orbits very often appears in the several branches of modern theory of dynamical systems, and, especially, the pseudo-orbit shadowing property usually plays an important role in the investigation of stability theory and ergodic theory as well as expansivity. Let $(X, d)$ be a compact metric space, and let $f: X \rightarrow X$ be a homeomorphism. For $\delta>0$, a sequence of points $\left\{x_{i}\right\}_{i=a}^{b} \subset X(-\infty \leq a<b \leq \infty)$ is called a $\delta$-pseudo-orbit of $f$ if $d\left(f\left(x_{i}\right), x_{i+1}\right)<\delta$ for all $a \leq i \leq b-1$.

Denote by $f_{\mid A}$ the restriction of $f$ to a subset $A$ of $X$. Let $\Lambda \subset X$ be a closed $f$-invariant set, that is, $f(\Lambda)=\Lambda$. We say that $f_{\mid \Lambda}$ has the shadowing property if for every $\epsilon>0$, there is $\delta>0$ such that for any $n \in \mathbb{N}$ and $\delta$-pseudo-orbit $\left\{x_{i}\right\}_{i=0}^{n-1} \subset \Lambda$ of $f$, there is $y \in X \epsilon$-shadowing the pseudo-orbit, that is, $d\left(f^{i}(y), x_{i}\right)<\epsilon$ for all $0 \leq i \leq n-1$. Notice that only $\delta$-pseudo-orbits of $f$ "contained in $\Lambda^{\text {" can be }}$ $\epsilon$-shadowed, but shadowing point $y \in X$ is "not necessarily" contained in $\Lambda$. We say that $f$ has the shadowing property if $X=\Lambda$ in the above definition. Since $X$ is compact, it is not hard to show that if $f_{\mid \Lambda}$ has the shadowing property, then every pseudo-orbit $\left\{x_{i}\right\}_{i=-\infty}^{\infty} \subset \Lambda$ can be shadowed by some true orbit.

The notion of expansivity has been intensively studied by several researchers, mainly from the topological view point, and lots of important fruitful results were obtained. Nowadays, expansive theory of dynamical systems has been well developed in both of the geometric theory and ergodic theory of dynamical systems as well as that of shadowing theory. Recently, the notion of the expansive measures was introduced by Morales and Sirvent [1] as a generalization of the notion of expansivity from the measure theoretical view point, and they exhibit the effectiveness of the use of it in expansive theory of dynamical systems (see [2-4] among others).

In this paper, inspired by the work of Morales and Sirvent, we introduce the notion of shadowable measures for a homeomorphism of a compact metric space as a generalization of the shadowing property from the measure theoretical view point, and consider the set of diffeomorphisms of a closed differentiable manifolds possessing the shadowable measures. The notion of such kind of measures is recently introduced by Lee and Morales [2], and they consider the shadowability of pseudo-orbits of a dynamical system from the measure theoretical view point. In our opinion, however, the introduced measure does not properly reflect the behavior of pseudo-orbits of the system. In fact, for a given 
pseudo-orbit $\left\{x_{i}\right\}_{i=0}^{\infty}$ of the system, they measure only the initial point $x_{0}$ of the pseudo-orbit, whole of the pseudo-orbit is not considered (see ([2], Definition 2.5)).

Recall that $(X, d)$ is a compact metric space and $f: X \rightarrow X$ is a homeomorphism of $X$. Let $X^{n}=X \times \cdots \times X$ (the $n$-times of direct product) be the sequences of points of $X$ with length $n(n \in \mathbb{N})$, and denote by $\mathcal{M}(X)$ the space of Borel probability measures of $X$. For any $\mu \in \mathcal{M}(X)$ (which is not necessarily $f$-invariant), let $\mu_{n}=\mu \times \cdots \times \mu$ (n-times) be the direct product measure of $X^{n}$. For any $\delta>0$, denote by $\mathcal{P O}(\delta, n)$ the space of $\delta$-pseudo-orbits $\left\{x_{i}\right\}_{i=0}^{n-1} \in X^{n}$ of $f$, and for $\epsilon>0$, denote by $\mathcal{S P O}(\delta, \epsilon, n)(\subset \mathcal{P O}(\delta, n))$ the set of $\delta$-pseudo-orbits $\epsilon$-shadowed by some point.

We say that $\mu \in \mathcal{M}(X)$ is a shadowable measure of $f$ (or simply, $f$ is $\mu$-shadowable) if for any $\epsilon>0$, there exists $\delta>0$ such that

$$
\mu_{n}(\mathcal{S P O}(\delta, \epsilon, n))=\mu_{n}(\mathcal{P O}(\delta, n))
$$

for any $n \in \mathbb{N}$ (if $A$ is a subset of $X$, then we define the shadowable measure for $f_{\mid A}$ by the same manner). Observe that if $f: X \rightarrow X$ has the shadowing property, then $f$ is $\mu$-shadowable for any $\mu \in \mathcal{M}(X)$. Denote by $\operatorname{supp}(\mu)$ the support of $\mu \in \mathcal{M}(X)$. Then, since $X$ is compact, we can see that if $f$ is $\mu$-shadowable and $f(\operatorname{supp}(\mu))=\operatorname{supp}(\mu)$, then $f: \operatorname{supp}(\mu) \rightarrow \operatorname{supp}(\mu)$ has the shadowing property (see Lemma 1 in Section 3).

Let $M$ be a closed $C^{\infty}$ manifold, and let $\operatorname{Diff}(M)$ be the space of diffeomorphisms of $M$ endowed with the $C^{1}$-topology. In this paper, we denote by $\mathcal{S}$ the set of $f \in \operatorname{Diff}(M)$ possessing the shadowing property. It is well-known that $f \in \operatorname{int} \mathcal{S}$ if and only if $f$ satisfies both Axiom A and the strong transversality condition (see [5-7] among others), where int means the $C^{1}$-interior of the set.

Hereafter, let $P(f)$ be the set of periodic points of $f \in \operatorname{Diff}(M)$, and let $\Omega(f)$ be the set of non-wandering points of it. Let $\Lambda \subset M$ be a closed $f$-invariant set. Recall that $\Lambda$ is hyperbolic if the tangent bundle $T_{\Lambda} M$ has a $D f$-invariant splitting $E^{S} \oplus E^{u}$ with constants $C>0$ and $0<\lambda<1$ such that

$$
\left\|\left.D f^{n}\right|_{E_{x}^{s}}\right\| \leq C \lambda^{n} \text { and }\left\|\left.D f^{-n}\right|_{E_{x}^{u}}\right\| \leq C \lambda^{n}
$$

for all $x \in \Lambda$ and $n \geq 0$. It is well-known that if $\Lambda$ is hyperbolic, then $f_{\mid \Lambda}$ has the shadowing property (see [8]). Recall that the shadowing point is not necessarily contained in $\Lambda$.

We say that $f$ satisfies Axiom $A$ if $\Omega(f)$ is hyperbolic and $\Omega(f)=\overline{P(f)}$. The stable manifold of a point $x$ is the set

$$
W^{s}(x)=\left\{y \in M: d\left(f^{n}(x), f^{n}(y)\right) \rightarrow 0 \text { as } n \rightarrow \infty\right\} .
$$

The unstable manifold $W^{u}(x)$ of $x$ is also defined analogously for $n \rightarrow-\infty$. It is well-known that if $\Lambda$ is hyperbolic, then $W^{s}(x)$ and $W^{u}(x)$ are both immersed manifolds for each $x \in \Lambda$ (for instance, see [8]). Let $f$ satisfies Axiom A. We say that $f$ satisfies the strong transversality condition if all the stable and the unstable manifolds are transverse at any point of their intersection.

To describe our results, let us introduce some notation on probability measures of $M$. Let us endow $\mathcal{M}(M)$ with the weak topology. It is well-known that the set of $f$-invariant measures, $\mathcal{M}_{f}(M)$, is a non-empty compact subset of $\mathcal{M}(M)$. We say that $\mu \in \mathcal{M}(M)$ is atomic if there exists a point $x \in M$ such that $\mu(\{x\})>0$ (denote by $\mathcal{A}(M)$ the set of atomic measures of $M$ ). It is known that the set of non-atomic measures is a residual set in $\mathcal{M}(M)$ (see [9]).

We define two subsets $\mathcal{P S}$ and $\mathcal{P} \mathcal{I} \mathcal{S}$ of $\operatorname{Diff}(M)$ as follows from a measure-theoretical view point and characterize them in the context of geometric theory of differentiable dynamical systems.

$\mathcal{P S}=\{f \in \operatorname{Diff}(M): f$ is $\mu$-shadowable for any $\mu \in \mathcal{M}(M) \backslash \mathcal{A}(M)\}$, and

$\mathcal{P} \mathcal{I} \mathcal{S}=\left\{f \in \operatorname{Diff}(M): f\right.$ is $\mu$-shadowable for any $\left.\mu \in \mathcal{M}_{f}(M) \backslash \mathcal{A}(M)\right\}$,

Recall that if $f \in \mathcal{S}$, then $f$ is $\mu$-shadowable for any $\mu \in \mathcal{M}(M) \backslash \mathcal{A}(M)$. Thus

$$
\mathcal{S} \subset \mathcal{P} \mathcal{S} \subset \mathcal{P} \mathcal{I} \mathcal{S}
$$

In this paper, we prove the following two theorems. 
Theorem 1. $f \in \operatorname{int} \mathcal{P S}$ if and only if $f$ satisfies both Axiom $A$ and the strong transversality condition.

The proof is based on the main result of [7], and the result is obtained by proving that int $\mathcal{P} \mathcal{S} \subset$ $\operatorname{int} \mathcal{S}$ (see Section 3).

Denote by $\mathcal{F}^{1}(M)$ the set of $f \in \operatorname{Diff}(M)$ such that there is a $C^{1}$-neighborhood $\mathcal{U}(f)$ of $f$ with property that every $p \in P(g)(g \in \mathcal{U}(f))$ is hyperbolic. It is proved by Hayashi [10] that $f \in \mathcal{F}^{1}(M)$ if and only if $f$ satisfies both Axiom A and no-cycle condition. The proof of the second result is based on Hayashi, and the result is obtained by proving that int $\mathcal{P} \mathcal{I} \subset \subset \mathcal{F}^{1}(M)$ (see Section 4).

Theorem 2. $f \in \operatorname{int} \mathcal{P I S}$ if and only if $f$ satisfies both Axiom $A$ and the no-cycle condition.

Owing to the above two theorems, the relationship between uniform hyperbolicity and $C^{1}$-robustness of the existence of shadowable measures turns out to be clear.

\section{Examples}

Recall that if a dynamical system has the shadowing property, then any probability mesasure is shadowable. The dynamical systems considered in this section do not have the shadowing property. First two examples have a measure that is not shadowable, but the last example has a shadowable measure.

Example 1. (The identity map of the unit interval).

Let $I$ be the unit interval $[0,1]$, and let $i d_{I}: I \rightarrow I$ be the identity map, that is, $i d_{I}(x)=x$ for $x \in I$. It is well-known that the identity map of $I$ does not have the shadowing property. Let $\mu$ be the Lebesgue probability measure on $I$. Then we can see that there exists $\epsilon_{0}>0$ such that for any $n \in \mathbb{N}$ and $\delta>0$, there is a subset

$$
\Gamma_{\delta}^{n} \subset \mathcal{P O}(\delta, n)
$$

with the property that $\mu_{n}\left(\Gamma_{\delta}^{n}\right)>0$ and non of any element in $\Gamma_{\delta}^{n}$ can be $\epsilon_{0}$-shadowed.

For any integer $m>0$ and $0 \leq i \leq 3 m-1$, denote by $I_{i}^{m}=\left[\frac{i}{3 m}, \frac{i+1}{3 m}\right]$. First we put $J_{i}^{m}=I_{i}^{m}$ for $0 \leq i \leq 3 m-1$, and then set $J_{i}^{m}=I_{3 m-1}^{m}$ for $i \geq 3 m$. It is easy to see that for any $x_{i} \in J_{i}^{m}(i \geq 0)$,

$$
d\left(i d_{I}\left(x_{i}\right), x_{i+1}\right)=d\left(x_{i}, x_{i+1}\right) \leq \frac{2}{3 m}<\frac{1}{m}
$$

so that $\left\{x_{i}\right\}_{i=0}^{\infty}$ is a $\frac{1}{m}$-pseudo-orbit of $i d_{I}$. Set

$$
\Gamma_{\delta}^{n}=\prod_{i=0}^{n-1} J_{i}^{m} \subset X^{n}
$$

Then

$$
\mu_{n}\left(\mathcal{P O}\left(\frac{1}{m}, n\right)\right) \geq \mu_{n}\left(\Gamma_{\delta}^{n}\right)=\mu_{n}\left(\prod_{i=0}^{n-1}\left|J_{i}^{m}\right|\right)=\left(\frac{1}{3 m}\right)^{n}>0
$$

for $n>0$.

Since $i d_{I}$ is the identity map, it is not hard to show that there exists $\epsilon_{0}>0$ (say, $\epsilon_{0}=1 / 3$ ) such that for any $\frac{1}{m}$-pseudo-orbit $\left\{x_{i}\right\}_{i=0}^{\infty}(m \gg 0)$ of $i d_{I}$, there is $n$ such that $\left\{x_{i}\right\}_{i=0}^{n-1}$ cannot be $\epsilon_{0}$-shadowed by any point $y \in I$.

Example 2. (Rotation maps of the unit circle). 
Let $S^{1}=\left\{e^{2 \pi i \theta}: \theta \in \mathbb{R}\right\} \subset \mathbb{C}$, and define a metric on $S^{1}$ by

$$
d\left(e^{2 \pi i \theta_{1}}, e^{2 \pi i \theta_{2}}\right)=\min \left\{\left|\theta_{1}-\theta_{2}\right|, 1-\left|\theta_{1}-\theta_{2}\right|\right\}
$$

For $\alpha \in \mathbb{R}$, let $\rho_{\alpha}: S^{1} \rightarrow S^{1}$ be a rotation map defined by $\rho_{\alpha}\left(e^{2 \pi i \theta}\right)=e^{2 \pi i(\alpha+\theta)}$.

Then the map $\rho_{\alpha}$ does not have the shadowing property. In fact, the identity map $i d_{S^{1}}$ does not have the shadowing property. For any (small) $\delta>0$, put $x_{j}=e^{2 \pi i j \delta}(j \in \mathbb{N})$. Then since

$$
d\left(i d_{S^{1}}\left(x_{j}\right), x_{j+1}\right)=d\left(x_{j}, x_{j+1}\right)=d\left(e^{2 \pi i j \delta}, e^{2 \pi i(j+1) \delta}\right)=\delta
$$

for any $j \in \mathbb{N}$, the sequence $\left\{x_{j}\right\}_{j=0}^{\infty}$ is a $\delta$-pseudo-orbit of $i d_{S^{1}}$. It is easy to see that the pseudo-orbit cannot be $\frac{1}{3}$-shadowed by $i d_{S^{1}}$-orbit for any $y \in S^{1}$.

Observe that if $\left|\theta_{1}-\theta_{2}\right|$ is small enough, then

$$
d\left(\rho_{\alpha}\left(e^{2 \pi i \theta_{1}}\right), \rho_{\alpha}\left(e^{2 \pi i \theta_{2}}\right)\right)=d\left(e^{2 \pi i\left(\alpha+\theta_{1}\right)}, e^{2 \pi i\left(\alpha+\theta_{2}\right)}\right)=\left|\theta_{1}-\theta_{2}\right| .
$$

Thus, it is not hard to show that the rotation map does not have the shadowing property as in the case $i d_{S^{1}}$.

Let $\mu$ be the usual Lebesgue probability measure on $S^{1}$ so that $\mu$ is $\rho_{\alpha}$-invariant. Then, as in the case of the identity map of the unit interval $I$, the measure of the set of non-shadowable pseudo-orbits is positive.

As stated before, the last example does not have the shadowing property, but has a shadowable measure. For the description, we need some notations.

Let $M^{2}$ be a surface, and let $f \in \operatorname{Diff}\left(M^{2}\right)$ satisfy Axiom A. The so-called $C^{0}$-transversality condition for $f$ is introduced by [11]. Roughly speaking, we say that the stable manifold $W^{s}(z)$ and the unstable manifold $W^{u}(z)$ of $z \in M^{2}$ are $C^{0}$-transverse at $z$ if these sets cross at $z$ as the graph of the function $y=x^{3}$ crosses the $x$-axis at the origin in the $x y$-plane. We say that $f$ satisfies the $C^{0}$-transversality condition if all the stable and the unstable manifolds are $C^{0}$-transverse at any point of their intersection. It is proved therein that $f$ satisfies the $C^{0}$-transversality condition if and only if $f$ has the shadowing property.

Example 3. (Axiom A diffeomorphisms with no-cycles).

We represent two-torus $\mathbb{T}^{2}$ as the square $[-2,2] \times[-2,2]$ with identified opposite sides in the Euclidean space $\mathbb{R}^{2}$. Let $g: \mathbb{T}^{2} \rightarrow \mathbb{T}^{2}$ be a diffeomorphism with the following properties (see Figure 1):

(1) the non-wandering set $\Omega(g)$ of $g$ is the union of 4 hyperbolic fixed points, that is, $\Omega(g)=$ $\left\{p_{1}, p_{2}, p_{3}, p_{4}\right\}$, where $p_{1}$ is a source, $p_{4}$ is a sink, and $p_{2}, p_{3}$ are saddles;

(2) with respect to coordinates $(v, w) \in[-2,2] \times[-2,2]$, the following conditions hold:

(2.1) $p_{1}=(1,2), p_{2}=(1,0), p_{3}=(-1,0), p_{4}=(-1,2)$,

(2.2) $W^{u}\left(p_{2}\right) \cup\left\{p_{3}\right\}=W^{s}\left(p_{3}\right) \cup\left\{p_{2}\right\}=[-2,2] \times\{0\} ，$

$$
W^{s}\left(p_{2}\right)=\{1\} \times(-2,2), W^{u}\left(p_{3}\right)=\{-1\} \times(-2,2),
$$

where $W^{s}\left(p_{i}\right)$ and $W^{u}\left(p_{i}\right)$ are the stable and unstable manifolds $(i=2,3)$, respectively, defined as usual;

(2.3) there exist neighborhoods $V_{2}, V_{3}$ of $p_{2}, p_{3}$ such that

$$
g(x)=p_{i}+D_{p_{i}} g\left(x-p_{i}\right) \text { if } x \in V_{i}
$$


(2.4) there exists a neighborhood $V$ of the point $O=(0,0)$ such that $g(V) \subset V_{3}, g^{-1}(V) \subset V_{2}$ and $g^{-1}$ is affine on $g(V)$,

(2.5) the eigenvalues of $D_{p_{3}} g$ are $\mu, v$ with $\mu>1,0<v<1$, and the eigenvalues of $D_{p_{2}} g$ are $\lambda, \kappa$ with $0<\lambda<1, \kappa>1$.

Please note that $g$ satisfies both Axiom A and the no-cycle condition (i.e., it is $\Omega$-stable) but does not have the shadowing property. Indeed, since $W^{s}\left(p_{3}\right) \backslash\left\{p_{3}\right\}=W^{u}\left(p_{2}\right) \backslash\left\{p_{2}\right\}$, the stable manifold and the unstable manifold are not $C^{0}$-transverse, and thus $g$ does not have the shadowing property. However, we show herewith that there exists shadowable measure for $g$.

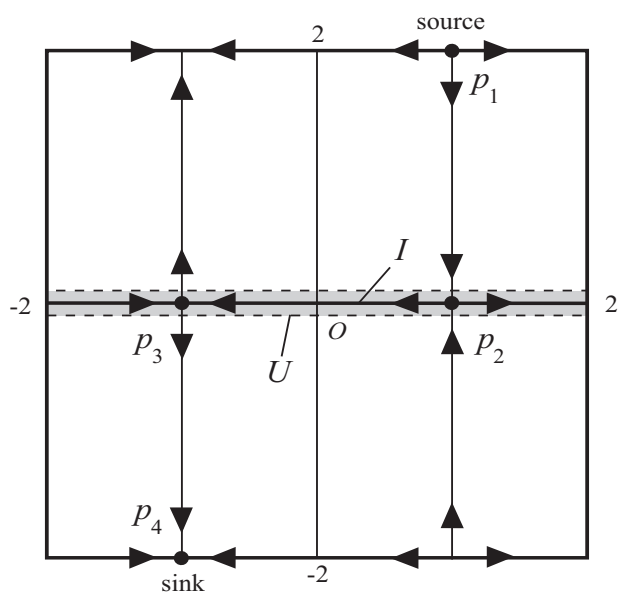

Figure 1. The structure of the stable and unstable manifolds.

Indeed, put $I=[-2,2] \times\{0\}$ and let $U$ be a small neighborhood of $I$ (see Figure 1). Define $v$ as the Lebesgue measure on $\mathbb{T}^{2} \backslash U$ such that $v\left(\mathbb{T}^{2}\right)=1$ and $v(U)=0$. Observe that for any $\delta>0$ and $n \in \mathbb{N}$, we can construct a $\delta$-pseudo-orbit $\left\{x_{i}\right\}_{i=0}^{n-1}$ of $g$ starting at a neighborhood of $p_{1}$, passing through $I$, and arriving at a neighborhood of $p_{4}$. It is easy to see that there exists $\epsilon>0$ such that for any $\delta>0$, there exists this kind of $\delta$-pseudo-orbit $\left\{x_{i}\right\}_{i=0}^{n-1}$ which cannot be $\epsilon$-shadowed by any orbit of $g$. Since $v(U)=0$, the $v_{n}$-measure of the set of such a kind of $\delta$-pseudo-orbits is 0 .

On the other hand, since $g$ is, in a sense, hyperbolic in the outside of $U$, we can see that any pseudo-orbit can be shadowed by an orbit of $g$. For, since $p_{4}$ is a sink fixed point, there is a neighbourhood $W\left(p_{4}\right)$ of $p_{4}$ and $0<\eta<1$ such that for any $x, y \in W\left(p_{4}\right), d(g(x), g(y)) \leq \eta d(x, y)$. For any $\epsilon>0$, choose $\delta>0$ small enough so that $\delta<\epsilon(1-\eta)$. Let $\left\{x_{i}\right\}_{i=0}^{n-1}$ be a $\delta$-pseudo-orbit of $g$ contained in $W\left(p_{4}\right)$. If $d\left(y, x_{0}\right)<\epsilon$ for some $y \in M$, then

$$
d\left(g(y), x_{1}\right) \leq d\left(g(y), g\left(x_{0}\right)\right)+d\left(g\left(x_{0}\right), x_{1}\right)<\epsilon \eta+\delta<\epsilon
$$

by the choice of $\delta$. Moreover, since $d\left(g\left(x_{1}\right), x_{2}\right)<\delta$, we have

$$
d\left(g^{2}(y), x_{2}\right) \leq d\left(g(g(y)), g\left(x_{1}\right)\right)+d\left(g\left(x_{1}\right), x_{2}\right)<\epsilon \eta+\delta<\epsilon .
$$

By continuing this manner, we can see that $d\left(g^{i}(y), x_{i}\right)<\epsilon$ for any $0 \leq i \leq n-1$. Thus, we have the following.

Claim 1. For any $\epsilon>0$, there is $\delta>0$ such that any $\delta$-pseudo-orbit $\left\{x_{i}\right\}_{i=0}^{n-1}$ of $g$ which is contained in $W\left(p_{4}\right)$ can be $\epsilon$-shadowed by some point.

Observe that since $p_{1}$ is a source fixed point, a similar property also holds in a neighbourhoof $W\left(p_{1}\right)$ of $p_{1}$ with respect to $g^{-1}$. The assertion of the following claim can be easily checked. 
Claim 2. There are a constant $N>0$ and $\delta_{0}>0$ such that for any $\delta$-pseudo-orbit $\left\{x_{i}\right\}_{i=0}^{n-1}$ of $g\left(0<\delta<\delta_{0}\right)$, if

$$
\left\{x_{i}\right\}_{i=0}^{n-1} \cap\left\{W\left(p_{4}\right) \cup W\left(p_{1}\right) \cup U\right\}=\varnothing,
$$

then $n \leq N$.

It is not hard to show that for any $\epsilon>0$, there exists $0<\delta_{1}<\delta_{0}$ such that any $\delta$-pseudo-orbit $\left\{x_{i}\right\}_{i=0}^{n-1}$ of $g\left(0<\delta<\delta_{1}\right)$ with $n \leq N$ can be $\epsilon$-shadowed some point. Combining this fact with the assertion of Claim 1, we can see that the map $g$ has the shadowing property in the outside of $U$. Thus $v$ is a shadowable measure.

\section{Proof of Theorem 1}

In this section, we give a proof of Theorem A. Let $f \in \operatorname{Diff}(M)$. As we explained before, it has shown that $f \in \operatorname{int} \mathcal{S}$ if and only if $f$ satisfies both Axiom A and the strong transversality condition. Thus, to get the conclusion, it is enough to show that int $\mathcal{P} \mathcal{S} \subset$ int $\mathcal{S}$.

In the following lemma, let $(X, d)$ be a compact metric space, and let $f: X \rightarrow X$ be a homeomorphism.

Lemma 1. Let $\mu \in \mathcal{M}(X)$. If $f$ is $\mu$-shadowable, then for any $\epsilon>0$, there exist $\delta>0$ such that for any $n \geq 1$ and $\delta$-pseudo-orbit $\left\{x_{i}\right\}_{i=0}^{n-1} \subset \operatorname{supp}(\mu)$ of $f$, there is $y \in X \in$-shadowing the pseudo-orbit.

Proof. Since $\mu$ is shadowable, for any $\epsilon>0$, there exists a $\delta>0$ such that for any $n \in \mathbb{N}$,

$$
\mu_{n}\left(\mathcal{S P O}\left(2 \delta, \frac{\epsilon}{2}, n\right)\right)=\mu_{n}(\mathcal{P O O}(2 \delta, n))
$$

Fix $n$ and let a $\delta$-pseudo-orbit $\left\{x_{i}\right\}_{i=0}^{n-1} \subset \operatorname{supp}(\mu)$ of $f$ be given. Then there exists $y \in X$ such that $d\left(f^{i}(y), x_{i}\right)<\epsilon$ for $0 \leq i \leq n-1$. Indeed, choose $0<r<\min \left\{\frac{\delta}{2}, \frac{\epsilon}{2}\right\}$ such that $d(x, y)<r$ implies $d(f(x), f(y))<\frac{\delta}{2}$, and put

$$
W_{r}^{n}=\prod_{i=0}^{n-1} B_{r}\left(x_{i}\right),
$$

where $B_{r}(x)=\{y \in X: d(x, y)<r\}$. Then we have $\mu_{n}\left(W_{r}^{n}\right)>0$ since $\left\{x_{i}\right\}_{i=0}^{n-1} \subset \operatorname{supp}(\mu)$. For any sequence $\left\{x_{i}^{\prime}\right\}_{i=0}^{n-1} \in W_{r}^{n} \subset X^{n}$ we have

$$
\begin{aligned}
d\left(f\left(x_{i}^{\prime}\right), x_{i+1}^{\prime}\right) & \leq d\left(f\left(x_{i}^{\prime}\right), f\left(x_{i}\right)\right)+d\left(f\left(x_{i}\right), x_{i+1}\right)+d\left(x_{i+1}, x_{i+1}^{\prime}\right) \\
& <\frac{\delta}{2}+\delta+r<2 \delta
\end{aligned}
$$

and so $\left\{x_{i}^{\prime}\right\}_{i=0}^{n-1}$ is a $2 \delta$-pseudo-orbit of $f$. Thus, there is $y \in X$ such that

$$
d\left(f^{i}(y), x_{i}^{\prime}\right)<\frac{\epsilon}{2}(0 \leq i \leq n-1)
$$

for some $\left\{x_{i}^{\prime}\right\}_{i=0}^{n-1}$ in $W_{r}^{n}$ since $\mu$ is a shadowable measure, and thus we have

$$
d\left(f(y), x_{i}\right)=d\left(f(y), x_{i}^{\prime}\right)+d\left(x_{i}^{\prime}, x_{i}\right)<\epsilon(0 \leq i \leq n-1) .
$$

End of the proof of Theorem 1. Suppose $f \in \operatorname{int} \mathcal{P} \mathcal{S}$, and let $\mu_{L}$ be the Lebesgue measure on $M$. Then, since $\operatorname{supp}\left(\mu_{L}\right)=M$, the proof of Theorem 1 quickly follows from Lemma 1 , so that Theorem 1 is proved. 


\section{Proof of Theorem 2}

In what follows, we give a proof of Theorem 2. Recall that $f \in \mathcal{F}^{1}(M)$ if and only if $f$ satisfies both Axiom A and no-cycle condition by [10]. Thus, to get the conclusion, it is enough to show that $\operatorname{int} \mathcal{P} \mathcal{I} \mathcal{S} \subset \mathcal{F}^{1}(M)$. Suppose $f \in \operatorname{int} \mathcal{P} \mathcal{I} \mathcal{S}$. The existence of the non-hyperbolic periodic point of $f$ easily gives a small periodic curve with period $l$ for some $\varphi C^{1}$-nearby $f$ such that the restriction of the map $\varphi^{l}$ to the curve is the identity map (see End of the proof of Theorem 2 below). From this, we will show that periodic points of any $g, C^{1}$-nearby $f$, are all hyperbolic. This kind of result was first obtained by the first author in ([12], Proposition 1). To show this fact we use the next lemma several times.

Lemma 2. Let $f \in \operatorname{Diff}(M)$ and let $\mathcal{U}(f)$ be given. Then there is $\delta>0$ such that for a finite set $\left\{x_{1}, x_{2}, \cdots, x_{N}\right\}$, a neighborhood $U$ of $\left\{x_{1}, x_{2}, \cdots, x_{N}\right\}$ and linear maps $L_{i}: T_{x_{i}} M \rightarrow T_{f\left(x_{i}\right)} M$ satisfying $\left\|L_{i}-D_{x_{i}} f\right\| \leq \delta$ for all $1 \leq i \leq N$, there are $\epsilon_{0}>0$ and $g \in \mathcal{U}(f)$ such that

(a) $g(x)=f(x)$ if $x \in M \backslash U$, and

(b) $g(x)=\exp _{f\left(x_{i}\right)} \circ L_{i} \circ \exp _{x_{i}}^{-1}(x)$ if $x \in B_{\epsilon_{0}}\left(x_{i}\right)$ for all $1 \leq i \leq N$.

Observe that the assertion $(b)$ implies that

$$
g(x)=f(x) \text { if } x \in\left\{x_{1}, x_{2}, \cdots, x_{N}\right\}
$$

and that $D_{x_{i}} g=L_{i}$ for all $1 \leq i \leq N$. The proof is essentially contained in the proof of ([13], Lemma 1).

End of the proof of Theorem 2. Suppose $f \in \operatorname{int}(\mathcal{P} \mathcal{I} \mathcal{S})$, and we show that $f \in \mathcal{F}^{1}(M)$. By contradiction, assume that $f \notin \mathcal{F}^{1}(M)$ and we shall derive a contradiction. Since $f \in \operatorname{int}(\mathcal{P} \mathcal{I} \mathcal{S})$, there exists a $C^{1}$-neighborhood $\mathcal{U}(f)$ of $f$ such that for any $g \in \mathcal{U}(f)$ and any $\mu \in \mathcal{M}_{g}(M) \backslash \mathcal{A}(M)$, $g$ is $\mu$-shadowable. On the other hand, it follows from the assumption $\left(f \notin \mathcal{F}^{1}(M)\right)$ that there are $g \in \mathcal{U}(f)$ and non-hyperbolic periodic point $p$ of $g$.

At first, by Lemma 2, with a small modification of the map $g$ with respect to the $C^{1}$-topology, we may assume that $D_{p} g^{\pi(p)}$ has only one eigenvalue $\lambda$ with modulus equal to 1 (or only one pair of complex conjugated eigenvalues, see Case 2 below). Denote by $E_{p}^{c}$ the eigenspace corresponding to $\lambda$.

Case 1. $\operatorname{dim} E_{p}^{c}=1$.

In this case, we suppose further that $\lambda=1$ for simplicity (the other case is similar). Then, by Lemma 2, there are $\epsilon_{0}>0$ and $\varphi \in \mathcal{U}(f)$ such that $\varphi^{\pi(p)}(p)=g^{\pi(p)}(p)=p$ and

$$
\varphi(x)=\exp _{g^{i+1}(p)} \circ D_{g^{i}(p)} g \circ \exp _{g^{i}(p)}^{-1}(x)
$$

if $x \in B_{\epsilon_{0}}\left(g^{i}(p)\right)$ for $0 \leq i \leq \pi(p)-2$, and

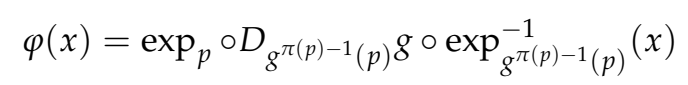

if $x \in B_{\epsilon_{0}}\left(g^{\pi(p)-1}(p)\right)$. We can choose $0<\epsilon_{1}<\epsilon_{0}$ such that

$$
\varphi^{i}\left(B_{\epsilon_{1}}(p)\right) \subset B_{\epsilon_{0}}\left(\varphi^{i}(p)\right)(0 \leq i \leq \pi(p))
$$

and

$$
\varphi^{i}\left(B_{\epsilon_{1}}(p)\right) \cap \varphi^{j}\left(B_{\epsilon_{1}}(p)\right)=\varnothing(0 \leq i \neq j \leq \pi(p)-1) .
$$

Define a small arc with its center at $p$ by

$$
\mathcal{L}_{p}^{c}=\exp _{p}\left(E_{p}^{c}\left(\epsilon_{1}\right)\right)
$$


Here $E_{p}^{c}\left(\epsilon_{1}\right)$ is the $\epsilon_{1}$-ball in $E_{p}^{c}$ centered at the origin $O_{p}$. Since the eigenvalue $\lambda$ of $\left.D_{p} g^{\pi(p)}\right|_{E_{p}^{c}}$ is 1 , we have

$$
\begin{aligned}
& \varphi^{i}\left(\mathcal{L}_{p}\right) \cap \varphi^{j}\left(\mathcal{L}_{p}\right)=\varnothing(0 \leq i \neq j \leq \pi(p)-1), \\
& \varphi^{\pi(p)}\left(\mathcal{L}_{p}\right)=\mathcal{L}_{p} \text {, and } \\
& \left.\varphi^{\pi(p)}\right|_{\mathcal{L}_{p}} \text { is the identity map. }
\end{aligned}
$$

Denote by $E_{p}^{s}$ the eigenspace corresponding to the eigenvalues of $D_{p} g^{\pi(p)}$ with modulus less than 1 , and by $E_{p}^{u}$ the eigenspace corresponding to the eigenvalues with modulus more than 1 . Then

$$
T_{p} M=E_{p}^{s} \oplus E_{p}^{c} \oplus E_{p}^{u}
$$

For $x \in B_{\epsilon_{0}}(p)$ we define

$$
\mathcal{L}_{x}^{s u}=\exp _{p}\left(\exp _{p}^{-1}(x)+E_{p}^{s} \oplus E_{p}^{u}\right) \cap B_{\epsilon_{0}}(p) .
$$

If we put $\epsilon_{2}=\tau \cdot \frac{\epsilon_{1}}{4}$ for $0<\tau<1$, then, we may assume

$$
\epsilon_{2}<\min \left\{d(y, z) \mid y \in \bigcup_{x \in B_{\epsilon_{2}}(p)} \mathcal{L}_{x}^{s u}, z \in \mathcal{L}_{p} \backslash B_{\frac{\epsilon_{1}}{4}}(p)\right\}\left(<\frac{\epsilon_{1}}{4}\right)
$$

by reducing $\tau$ if necessary.

Let $m_{\mathcal{L}_{p}}$ be a normalized Lebesgue measure on $\mathcal{L}_{p}$. We define $\mu \in \mathcal{M}_{\varphi}(M)$ by

$$
\mu(C)=\frac{1}{\pi(p)} \sum_{j=0}^{\pi(p)-1} m_{\mathcal{L}_{p}}\left(\varphi^{-j}\left(C \cap \varphi^{j}\left(\mathcal{L}_{p}\right)\right)\right)
$$

for any Borel set $C$ of $M$. Observe that $\mu \in \mathcal{M}_{\varphi}(M) \backslash \mathcal{A}(M)$.

Let $\delta>0$. By the continuity of $\varphi$, there exists $0<\delta_{1}<\epsilon_{2}$ such that $d(x, y)<\frac{\delta_{1}}{2}$ implies

$$
d\left(\varphi^{i}(x), \varphi^{i}(y)\right)<\frac{\delta}{2}
$$

for $x, y \in M$ and $0 \leq i \leq \pi(p)-1$. Choose an integer $m>0$ such that $\frac{\epsilon_{1}}{2}<m \delta_{1}<\frac{3 \epsilon_{1}}{4}$. Then there are points

$$
x_{0}, x_{1}, x_{2}, \cdots, x_{m-1} \in \mathcal{L}_{p}
$$

such that

(i) $x_{0}=p$,

(ii) $B_{\frac{\delta_{1}}{2}}\left(x_{k}\right) \cap B_{\frac{\delta_{1}}{2}}\left(x_{k+1}\right) \neq \varnothing$ for $0 \leq k \leq m-2$,

(iii) $d\left(x_{0}, x_{k}\right)<\frac{3 \epsilon_{1}}{4}(k=1,2, \cdots, m-1), d\left(x_{0}, x_{m-1}\right)>\frac{3 \epsilon_{1}}{8}$.

For any $0 \leq k \leq m-1$ and $0 \leq \ell \leq \pi(p)-1)$, we set

$$
I_{k \pi(p)+\ell}=\varphi^{\ell}\left(B_{\frac{\delta_{1}}{2}}\left(x_{k}\right) \cap \mathcal{L}_{p}\right) .
$$

The set $S_{m}$ is defined by

$$
S_{m}=\left\{\left\{y_{i}\right\} \in M^{m \pi(p)} \mid y_{i} \in I_{i}, 0 \leq i \leq m \pi(p)-1\right\} .
$$


Then it is easily checked that $S_{m} \subset \mathcal{P O}(\delta, m \pi(p))$ and $\mu_{m \pi(p)}\left(S_{m}\right)>0$. We show that

$$
\mathcal{S P O}\left(\delta, \frac{\epsilon_{2}}{2}, m \pi(p)\right) \cap S_{m}=\varnothing .
$$

Let $\left\{y_{i}\right\} \in S_{m}$ and $y \in M$ satisfy $d\left(\varphi^{i}(y), y_{i}\right)<\frac{\epsilon_{2}}{2}$ for $0 \leq i \leq m \pi(p)-1$. Since

$$
\begin{aligned}
d\left(\varphi^{k \pi(p)}(y), p\right) & \leq d\left(\varphi^{k \pi(p)}(y), y_{k \pi(p)}\right)+d\left(y_{k \pi(p)}, x_{k \pi(p)}\right)+d\left(x_{k \pi(p)}, p\right) \\
& <\frac{\epsilon_{1}}{8}+\frac{\delta_{1}}{2}+\frac{3 \epsilon_{1}}{4}<\epsilon_{1}
\end{aligned}
$$

we have $d\left(\varphi^{k \pi(p)+j}(y), \varphi^{j}(p)\right) \leq \varepsilon_{0}$ for $0 \leq k \leq m-1$ and $0 \leq j \leq \pi(p)-1$. If we put $\exp _{p}^{-1}(y)=$ $v^{s}+v^{c}+v^{u}, v^{\sigma} \in E_{p}^{\sigma}(\sigma=s, c, u)$, then we have

$$
\begin{aligned}
& \varphi^{(m-1) \pi(p)}(y) \\
= & \exp _{p}\left(D_{p} g^{(m-1) \pi(p)}\left(v^{u}\right)+D_{p} g^{(m-1) \pi(p)}\left(v^{c}\right)+D_{p} g^{(m-1) \pi(p)}\left(v^{s}\right)\right) \\
\in & \exp _{p}\left(v^{c}+E_{p}^{u} \oplus E_{p}^{s}\right) \cap B_{\epsilon_{0}}(p) \\
\subset & \bigcup_{x \in B_{\epsilon_{2}}(p)} \mathcal{L}_{x}^{s u} .
\end{aligned}
$$

Since

$$
d\left(p, y_{(m-1) \pi(p)}\right) \geq d\left(p, x_{m-1}\right)-d\left(x_{m-1}, y_{(m-1) \pi(p)}\right) \geq \frac{3 \epsilon_{1}}{8}-\frac{\epsilon_{1}}{8}=\frac{\epsilon_{1}}{4},
$$

we have $d\left(\varphi^{(m-1) \pi(p)}(y), y_{(m-1) \pi(p)}\right) \geq \epsilon_{2}$, which is a contradiction.

Therefore we have $\mathcal{S P O}\left(\delta, \frac{\epsilon_{2}}{2}, m \pi(p)\right) \cap S_{m}=\varnothing$. But this contradicts with $\varphi \in \mathcal{U}(f)$.

Case 2. $\operatorname{dim} E_{p}^{c}=2$.

In the proof of the second case, to avoid notational complexity, we consider only the case $g(p)=p$. As in the first case, by Lemma 2, there are $\epsilon_{0}>0$ and $\varphi \in \mathcal{U}(f)$ such that $\varphi(p)=g(p)=p$ and

$$
\varphi(x)=\exp _{g(p)} \circ D_{p} g \circ \exp _{p}^{-1}(x)
$$

if $x \in B_{\epsilon_{0}}(p)$. With a small modification of the map $D_{p} g$, we may suppose that there is $l>0$ (the minimum number) such that $D_{p} g^{l}(v)=v$ for any $v \in E_{p}^{c}\left(\epsilon_{0}\right)$ by Lemma 2 .

Take $v_{0} \in E_{p}^{c}\left(\epsilon_{0}\right)$ such that $\left\|v_{0}\right\|=\frac{\epsilon_{0}}{4}$, and set

$$
\mathcal{J}_{p}=\exp _{p}\left(\left\{t \cdot v_{0}: 1 \leq t \leq 1+\frac{\epsilon_{0}}{4}\right\}\right) .
$$

Then $\mathcal{J}_{p}$ is an arc such that

$$
\begin{aligned}
& \varphi^{i}\left(\mathcal{J}_{p}\right) \cap \varphi^{i}\left(\mathcal{J}_{p}\right)=\varnothing(0 \leq i \neq j \leq l-1), \\
& \varphi^{l}\left(\mathcal{J}_{p}\right)=\mathcal{J}_{p} \text {, and } \\
& \left.\varphi^{l}\right|_{\mathcal{J}_{p}} \text { is the identity map. }
\end{aligned}
$$

Let $m_{\mathcal{J}_{p}}$ be the normalized Lebesgue measure on $\mathcal{J}_{p}$ and set

$$
\mu(C)=\frac{1}{l} \sum_{j=0}^{l-1} m_{\mathcal{J}_{p}}\left(\varphi^{-j}\left(C \cap \varphi^{j}\left(\mathcal{J}_{p}\right)\right)\right)
$$

for a Borel set $C$.

Then $\mu \in \mathcal{M}_{\varphi}(M) \backslash \mathcal{A}(M)$. As in the first case, we can prove that $\varphi$ is not $\mu$-shadowable, which contradicts the fact that $\varphi \in \mathcal{U}(f)$. Thus, Theorem 2 is proved. 
Author Contributions: The three authors contributed equally to this work.

Acknowledgments: K.S. is supported by JSPS KAKENHI Grant Number 16K05167 and N.S. is supported by JSPS KAKENHI Grant Number 15K04902.

Conflicts of Interest: The authors declare no conflict of interest.

\section{References}

1. Morales, C.A.; Sirvent, V.F. Expansive measures. In IMPA Mathematical Publications 29, Colóquio Brasileiro de Matemática; Instituto Nacional de Matemática Pura e Aplicada: Rio de Janeiro, Brazil, 2013.

2. Lee, K.; Morales, C.A. Topological stability and pseudo-orbit tracing property for expansive measures. J. Differ. Eq. 2017, 262, 3467-3487. [CrossRef]

3. Pacifico, M.J.; Vieitez, J.L. On measure expansive diffeomorphisms. Proc. Amer. Math. Soc. 2015, 143, 811-819. [CrossRef]

4. Sakai, K.; Sumi, N.; Yamamoto, K. Measure-expansive diffeomorphisms. J. Math. Anal. Appl. 1994, 414, 546-552. [CrossRef]

5. Pilyugin, S.Y.; Sakai, K. Shadowing and Hyperbolicity; Lecture Notes in Mathematics; Springer: Berlin, Germany, 2017; Volume 2193.

6. Robinson, C. Stability theorems and hyperbolicity in dynamical systems. Rocky Mt. J. Math. 1977, 7, 425-437. [CrossRef]

7. Sakai, K. Pseudo orbit tracing property and strong transversality of diffeomorphisms on closed manifolds. Osaka J. Math. 1994, 31, 373-386.

8. Robinson, C. Dynamical Systems: Stability, Symbolic Dynamics, and Chaos, 2nd ed.; Studies in Advanced Mathematics; CRC Press: Boca Raton, FL, USA, 1999.

9. Denker, M.; Grillenberger, C.; Sigmund, K. Ergodic Theory on Compact Spaces; Lecture Notes in Math; Springer: Berlin, Germany, 1976; Volume 527.

10. Hayashi, S. Diffeomorphisms in $\mathcal{F}^{1}(M)$ satisfy Axiom A. Ergod. Th. Dynam. Sys. 1992, 12, $233-253$. [CrossRef]

11. Pilyugin, S.Y.; Sakai, K. $C^{0}$ transversality and shadowing properties. Proc. Steklov Inst. Math. 2007, 256, $290-305$. [CrossRef]

12. Moriyasu, K. The topological stability of diffeomorphisms. Nagoya Math. J. 1991, 123, 91-102. [CrossRef]

13. Franks, J. Necessary conditions for stability of diffeomorphisms. Trans. Am. Math. Soc. 1971, 158, 301-308. [CrossRef]

(C) 2018 by the authors. Licensee MDPI, Basel, Switzerland. This article is an open access article distributed under the terms and conditions of the Creative Commons Attribution (CC BY) license (http:/ / creativecommons.org/licenses/by/4.0/). 